\title{
Manifestaciones cutáneas por COVID-19
}

\author{
DOI 10.5377/alerta.v4i2.10156
}

Karen Yesenia Gómez Romero ${ }^{{ }^{*}}$, Stefany Elizabeth Guerra Figueroa ${ }^{2}$

1. Departamento de ciencias fisiológicas, Universidad Evangélica de El Salvador, San Salvador, El Salvador. 2. Hospital General, Instituto Salvadoreño del Seguro Social, San Salvador, El Salvador.

${ }^{*}$ Correspondencia

$\square$ dra.karengomezromero@gmail.com

(1D) $0000-0002-5109-5762$

2. (1) 0000-0001-6349-1546

ACCESO ABIERTO

Cutaneous

Manifestations by

COVID-19

Citación recomendada:

Gómez Romero KY, Guerra

Figueroa SE, Manifestaciones

cutáneas por COVID-19.

Alerta. 2021:4(2):46-53

DOI 10.5377/alerta.v4i2.10156

\section{Recibido:}

08 de agosto 2020

\section{Aceptado:}

15 de febrero de 2021

\section{Publicado:}

21 de mayo de 2021

\section{Contribución de autoría:} KYGR': Análisis del caso, busqueda bibliográfica y redacción del manuscrito. SEGF²: Análisis del caso y redacción del manuscrito.

\section{Conflicto de intereses:}

Los autores declaran no tener conflictos de interés.

\begin{abstract}
Resumen
El estudio de las manifestaciones dermatológicas asociadas al Coronavirus tipo 2 del síndrome respiratorio agudo grave, causante de la pandemia de la enfermedad por Coronavirus 2019, contribuye al diagnóstico de COVID-19 en pacientes con manifestaciones cutáneas de evolución reciente asociados a sintomatología respiratoria o pacientes asintomáticos que han mantenido contacto estrecho con pacientes con infección por SARS-CoV-2 confirmada. La presente revisión tiene por objetivo describir los patrones dermatológicos más frecuentemente asociados a la COVID-19 de acuerdo con la literatura publicada, entre los que se destacan: erupciones maculopapulares, lesiones acrales de eritema-edema con vesículas o pústulas, erupciones vesiculares, erupciones tipo urticaria, lesiones tipo livedo o necrosis y petequias. Se realizó la búsqueda de la literatura en la base de datos PUBMED, utilizando los descriptores COVID 19 OR Coronavirus AND «skin manifestations». Se identificaron un total de 119 artículos publicados durante el año 2020 de los cuales se incluyeron 44 artículos referentes a reporte de casos y revisiones para el análisis, efectuándose finalmente un narrativo de los resultados. Se concluye que el conocimiento de las manifestaciones cutáneas asociadas a COVID-19 puede ayudar en el diagnóstico precoz y estratificación de riesgo.
\end{abstract}

\section{Palabras clave}

COVID 19, Coronavirus, Manifestaciones cutáneas.

\begin{abstract}
The study of the dermatological manifestations associated with the Severe Acute Respiratory Syndrome Coronavirus type 2, which causes the pandemic of the Coronavirus disease 2019 contributes to the diagnosis of COVID-19 in patients with recently developed skin manifestations associated with respiratory symptoms or asymptomatic patients who have maintained close contact with patients with confirmed SARS-CoV-2 infection. The present review aims to describe the dermatological patterns most frequently associated with COVID-19 according to the published literature, among which are: Maculopapular eruptions, acral erythema-edema lesions with vesicles or pustules, vesicular eruptions, urticarial-like eruptions, Livedo type lesions or necrosis, and petechiae. The literature search was carried out in the PUBMED database, using the descriptors COVID 19 OR Coronavirus AND «skin manifestations». A total of 119 articles published during 2020 were identified, of which 44 articles referring to case reports and reviews were included for analysis, finally making a narrative of the results. It is concluded that the knowledge of the cutaneous manifestations associated with COVID-19 can help in the early diagnosis and risk stratification.
\end{abstract}

Keywords

COVID-19 virus disease, Coronaviruses, Skin manifestation.

La COVID-19 es una enfermedad altamente contagiosa causada por el SARS-CoV-2, que fue reportado por primera vez el 1 de diciembre del año 2019 en la ciudad de Wuhan China. En marzo de 2020, la Organización Mundial de la Salud elevó el estado de la enfermedad de emergencia de salud pública de importancia internacional a pandemia'.

El SARS-CoV-2 es transmitido principalmente a través de gotitas respiratorias que contienen el virus o por objetos contamina- dos $^{2}$. El periodo de incubación varía entre 2 y 14 días, con un promedio de cinco días. Las personas que desarrollan la enfermedad presentan fiebre, tos, conjuntivitis, disgeusia, anosmia, náuseas, diarrea y malestar general ${ }^{3}$. Además, se ha reportado dificultad respiratoria, falla respiratoria aguda, trastornos de la coagulación, disfunción orgánica y complicaciones secundarias a una respuesta inflamatoria sistémica, las cuales se asocian a un aumento de la mortalidad ${ }^{4}$. 
Las manifestaciones cutáneas pueden formar parte del cuadro de una infección viral (por ejemplo: dengue, zika, sarampión, entre otros). En este sentido se han descrito diversos hallazgos en la piel de pacientes con COVID-19, entre estas se incluyen rash morbiliforme, lesiones maculopapulares, purpúricas, urticaria, lesiones acrales de aspecto livedoide, pseudosabañones y erupciones vesiculares ${ }^{5}$. Por ser una infección viral emergente, las manifestaciones cutáneas asociadas a SARS-CoV-2 son presentaciones clínicas a considerar. El creciente número de reporte de casos y de series clínicas que recientemente han sido publicados, han descrito un espectro de manifestaciones cutáneas asociadas a esta enfermedad; por lo que el objetivo de esta revisión es describir las principales manifestaciones cutáneas asociadas a la infección por la COVID-19 descritos en la literatura.

\section{Discusión temática}

Las manifestaciones cutáneas de la COVID-19, según los informes de la literatura, muestran una gran diversidad. Series de casos de todo el mundo han identificado una variedad de posibles manifestaciones dermatológicas de COVID-19. La frecuencia (que varía del 0,2 al 20,4 por ciento de los casos) y el momento de las manifestaciones cutáneas de COVID-19 son difíciles de determinar $^{5}$. Estas manifestaciones de infecciones virales en general pueden derivarse de inoculación directa del virus, diseminación o reactivación del virus desde otro sitio, interacción del virus con el sistema inmunológico, respuestas inmunocelulares y humorales relacionadas que incluyen la acción de linfocitos y anticuerpos específicos contra el virus ${ }^{6}$. A pesar de que la fisiopatología de las manifestaciones cutáneas en el contexto de COVID-19 es incierta, se ha demostrado que en la capa basal de la epidermis se encuentran receptores para la enzima convertidora de angiotensina 2, la cual es un receptor en la membrana de la célula huésped, con una alta afinidad por la proteína $S$ del SARS$\mathrm{CoV}-2^{7,8}$, siendo las diferentes manifestaciones dermatológicas en pacientes con COVID-19 el reflejo de la interacción del virus con la piel. Las respuestas inmunes hiperactivas, por activación del complemento o por injuria microvascular, se han descrito como otros posibles mecanismos fisiopatológicos que podrían explicar la presentación de manifestaciones cutáneas por COVID-199,10.

La aparición de las lesiones cutáneas en relación con la viremia y los síntomas generales, podría ayudar a comprender los mecanismos fisiopatológicos implicados en el desarrollo de lesiones en los pacientes con COVID-19. En el caso de que las lesiones cutáneas precedan a los síntomas generales o incluso sean el único signo de posible infección, podrían servir como indicadores tempranos de la enfermedad o como indicadores de portadores asintomáticos del virus. Según reportes también puede aparecer posterior al desarrollo de otros síntomas y en casos asintomáticos, leves, moderados y severos.

Por otro lado, se debe establecer el diagnóstico diferencial con otras enfermedades virales que presentan manifestaciones $\mathrm{Cu}$ táneas similares y de gran relevancia, sobre todo en Latinoamérica, como en el caso infecciones por dengue, zika y chikungunya. Los síntomas cutáneos que ocurren en etapas tardías de la infección o incluso después de la resolución de los síntomas principales, no implican una replicación per se del virus en el sistema respiratorio o probabilidad de contagio, ya que hoy en día se sabe que en pacientes con COVID-19 leve sin requerimiento de hospitalización por $\mathrm{UCl}$, después de 10 días de inicio de síntomas y con un mínimo de tres días sin síntomas, ya no es contagioso, independientemente del resultado de una RT-PCR por hisopado nasofaríngeo; pacientes asintomáticos posterior a 10 días de dar positivo a la prueba de SARSCoV-2 ${ }^{11}$. Así mismo, pacientes con criterios para hospitalización por UCl, a los 15 a 20 días posterior al inicio de síntomas, dejan de ser contagioso, independiente de si presenta o no síntomas cutáneos o en otros sistemas. Las secuelas posteriores a los lapsos descritos son secundarias a la tormenta de citoquinas y al tratamiento dado ${ }^{12}$.

Actualmente se están realizando esfuerzos para conocer la prevalencia de las manifestaciones cutáneas en el contexto de esta enfermedad, la relación temporal con otros hallazgos clínicos y la posibilidad de una enfermedad subyacente grave. Debido al continuo reporte de casos y realización de estudios centrados en la caracterización y clasificación de las lesiones vistas en pacientes con COVID-19, se han elaborado clasificaciones para describir los patrones clínicos de las manifestaciones cutáneas, su relación con aspectos demográficos, la severidad de la enfermedad, relación con los síntomas y su pronóstico ${ }^{13}$.

Recientemente en España se realizó la descripción de patrones morfológicos asociados con COVID-19 por medio de un método de consenso, en donde se incluyó el reporte de 375 pacientes con lesiones cutáneas de inicio reciente sin etiología clara de las mismas y con sospecha de enfermedad por SARS-COV-2 o su confirmación por labo- 
ratorio, independientemente de la presencia de signos o síntomas. Dicho consenso permitió la elaboración del primer estudio prospectivo para clasificar las manifestaciones cutáneas por COVID-19 dentro de 5 patrones clínicos que incluyen: lesiones acrales de eritema-edema con vesículas o pústulas (pseudosabañones), erupciones vesiculares, lesiones urticariales, lesiones maculopapulares y livedo o necrosis ${ }^{14}$. Otros autores han descrito las lesiones petequiales como un sexto patrón clínico a considerarse ${ }^{15}$.

Las lesiones dermatológicas evidenciadas pueden presentarse también en otras virosis; por ejemplo, el rash maculopapular, las lesiones urticariformes, petequias, equimosis, entre otras, que ocasionalmente acompañan al dengue, de especial interés para la región latinoamericana; Cabe destacar que se han documentado casos de coinfección dengue-COVID19 y se han reportado resultados falsos positivos en pruebas rápidas (IgM e lgG) para dengue, en casos confirmados de COVID-1916.

Debido a que cerca del $20 \%$ de los pacientes con COVID-19 desarrollan manifestaciones cutáneas, el reconocimiento y clasi- ficación de las mismas es necesario durante la evaluación física. Por ello, se ha formulado recientemente un algoritmo para facilitar la clasificación de lesiones cutáneas por COVID-19 que puede ser aplicado por médicos no dermatólogos durante la atención de pacientes con COVID-19 y manifestaciones cutáneas asociadas. Se propone la división de las lesiones en tres principales grupos: lesiones que no desaparecen con la presión, que incluyen el rash con petequias, lesiones isquémicas acrales y livedo reticularis; lesiones que desaparecen con la presión como rash urticarial y rash eritematoso; y el patrón vesículas o costras/erosiones ${ }^{17}$.

Los patrones clínicos de las manifestaciones cutáneas por COVID-19 descritos se han asociado a diversos grados de severidad de acuerdo con la evolución de la enfermedad, siguiendo un gradiente desde la enfermedad menos grave en pseudo-sabañones hasta la más grave en pacientes con presentación de lesiones livedoides y necrosis. El reconocimiento temprano de signos cutáneos que se asocian a una mayor gravedad es esencial para el manejo oportuno de complicaciones asociadas. (Figura 1)

Figura 1. Incidencia de patrones clínicos de manifestaciones cutáneas y su relación con la severidad de COVID-19.

Livedo o Necrosis (6\%)

Lesiones maculopapulares (47 \%)

Eritema y Urticaria (19\%)

Erupciones Vesiculares (9\%)

Lesiones acrales de eritema-edema con vesículas o pústulas (pseudo-sabañones) (19\%)

Fuente: Elaboración propia, con datos de incidencia tomados de: Casas CG, Català A, Hernández GC, RodríguezJiménez P, Fernández-Nieto D, Lario AR-V, et al. Classification of the cutaneous manifestations of COVID-19: a rapid prospective nationwide consensus study in Spain with 375 cases. British Journal of Dermatology. 2020; 183(1):71-7. 


\section{Lesiones acrales de eritema-edema con vesículas 0 pústulas (pseudosabañones)}

Las lesiones acrales de eritema-edema con vesículas o pústulas (pseudo-sabañones) o también conocidas como lesiones acroisquémicas, son lesiones que han sido documentadas con una prevalencia del $19 \%$ de acuerdo con el reporte de series de casos realizado en España ${ }^{14}$. Estas lesiones, usualmente unilaterales, en el contexto de la COVID-19 se presentan sin antecedentes de exposición al frío, afectan zonas acrales y las principales partes afectadas son los dedos de pies y manos. Morfológicamente se caracterizan por pápulas y maculas eritemato violáceas, con presencia de edema y posible evolución ampollosa; adicionalmente pueden acompañarse de dolor y prurito ${ }^{18}$. Las lesiones tienden a resolver espontáneamente en 1 a 4 semanas $^{19}$. El hallazgo clínico de pseudo-sabañones constituye una manifestación inusual de COVID-19 y se presentan principalmente en niños y adolescentes con sintomatología leve ${ }^{20}$, sugiriendo ser un marcador de respuesta antiviral eficaz por parte del huésped ${ }^{21}$.

Los pseudo-sabañones pueden aparecer sin otros síntomas de COVID-19 más comúnmente en la población general y característicamente aparecen con mayor frecuencia durante la evolución tardía de la enfermedad, por lo que podrían ser útiles como marcadores epidemiológicos ${ }^{14}$ o como un posible signo de infección por COVID-19 en pacientes pediátricos paucisintomáticos ${ }^{20}$.

Dentro de los mecanismos fisiopatológicos por el cual se originan estas lesiones se han descrito: trastornos de la coagulación que cursen con aumento de los productos de degradación del dímero $D$ y del fibrinógeno y mecanismos inmunológicos que proponen que la causa principal de las lesiones compatibles con perniosis o pseudo-sabañones es la respuesta temprana del interferón tipo I, la cual es una citoquina con función antiviral capaz de inhibir la liberación no controlada de citoquinas proinflamatorias ${ }^{22,23}$.

\section{Erupciones vesiculares}

El patrón vesicular consiste en la presentación de lesiones descritas en su mayoría como una erupción diseminada, que afecta principalmente tronco y extremidades, constituida por vesículas pruriginosas. Se han observado dos diferentes tipos de erupción vesicular: una erupción diseminada, polimorfa o de aspecto variceliforme, que constituye la forma de presentación de erupción vesicular más frecuente y una erupción vesicular localizada a nivel de tronco principalmente, en el mismo estadio de evolución o monomorfa ${ }^{24,25,26}$. Según reporte de casos, su incidencia en pacientes con COVID-19 ha sido del $9 \%{ }^{14}$.

Las lesiones vesiculares en el contexto de infección por SARS-CoV-2 se observan principalmente en etapas tempranas de la enfermedad y se asocian con la fase activa de la misma, tanto en niños como en adultos. Estas lesiones suelen presentarse en formas leves a moderadas de la enfermedad ${ }^{26}$. Se postula que los cambios histopatológicos referentes a degeneración vacuolar y apoptosis de queratinocitos que se presentan en pacientes con infección por SARS-CoV-2 y exantema variceliforme puede ser secundario a daño citopático directo por el virus sobre los queratinocitos ${ }^{20}$.

Entre los diagnósticos diferenciales, se deben incluir manifestaciones de herpes simple, eritema multiforme y enfermedades ampollosas autoinmunes. Es recomendable realizar test de Tzanck o PCR para varicela-zoster para descartar la enfermedad herpética, ya que además se han descrito reactivaciones de herpes en pacientes con COVID-1927.

\section{Exantema urticariforme}

El patrón de lesión similar a la urticaria se ha reportado con una incidencia del $19 \%$ en series de casos reportados en España ${ }^{14}$. Se ha descrito como una erupción cutánea eritematosa diseminada con predominio a nivel de cara, tronco y regiones acrales que respeta palmas y plantas, acompañado de prurito ${ }^{28}$. Las lesiones urticariales pueden preceder a los síntomas de infección por COVID-19, por lo que pueden fungir como pista de diagnóstico temprano $29,30,31$ o aparecer en conjunto con los síntomas sistémicos en las formas moderadas de la enfermedad ${ }^{27}$. A pesar de ello, la mayoría de las lesiones urticariales y maculopapulares pueden no ser muy útiles para el diagnóstico de COVID-19, ya que pueden tener muchas otras causas. Dentro de estas, las reacciones farmacológicas deben ser un diagnóstico diferencial importante a considerar ${ }^{31}$. No obstante, los pacientes con lesiones urticariales deben ser evaluados cuidadosamente por la posibilidad de infección por SARS-CoV2, y por la posibilidad de complicaciones asociadas como angioedema ${ }^{30}$.

\section{Lesiones maculopapulares}

Las lesiones maculopapulares son uno de los patrones dermatológicos que se asocian con 
más frecuencia a COVID-19, ya que ha sido reportado con una incidencia de $47 \%$ en series de casos. Se caracteriza por una erupción de distribución cefalocaudal, constituida por maculas y pápulas eritematosas, perifoliculares en algunos casos, que se puede acompañar de descamación en etapas tardías de la evolución de las lesiones y, además, pueden presentar un aspecto pseudovesicular ${ }^{14}$. La erupción maculopapular se presenta de manera simultánea con otros síntomas por COVID-19y, en menor frecuencia, posterior a los mismos $^{31}$. De igual forma, las lesiones maculopapulares se han visto asociadas a enfermedad por COVID-19 de evolución moderada a grave y se han reportado en pacientes pediátricos y adultos ${ }^{32}$. En el contexto de la COVID-19, el efecto citopático directo sobre los queratinocitos puede explicar la presentación de este patrón de lesión ${ }^{18}$.

Las lesiones maculopapulares también han sido descritas con una morfología similar a la pitiriasis rosada con presentación típica de inicio, con una placa primaria eritematosa con descamación fina o medallón heráldico, que evoluciona con el desarrollo de lesiones diseminadas más pequeñas ${ }^{33,34}$. Esto podría deberse a la reactivación del virus herpes humano 6 a causa de COVID-1935.

El exantema maculopapular es considerado como el exantema típico de las infecciones virales como dengue, zika, sarampión, entre otros, debido a la activación de citocinas $^{36}$. O puede observarse como una manifestación cutánea por reacciones alérgicas a medicamentos; por lo que, para definir con precisión la causa del mismo, es importante recopilar la mayor cantidad de información posible sobre el episodio, la cronología y la exposición previa a fármacos. Por ello, una anamnesis completa, exploración física y debido a la alta sospecha de COVID-19, la realización de una prueba de RT-PCR confirmatoria es necesario para el diagnóstico de infección por SARS-CoV 2 frente a otros virus endémicos ${ }^{37}$.

\section{Livedo o necrosis}

El patrón livedo reticularis o necrosis es un patrón vascular que pone en evidencia las alteraciones en la coagulación y el daño vascular con que ha estado relacionada la COVID-19, en el que eventualmente pueden desencadenarse estados protrombóticos, los cuales pueden desarrollarse secundario a la producción de interleucina 6 (IL-6), que puede estar involucrada en la patogenia de la trombosis vascular a través de sus efectos sobre la agregación y activación plaquetaria o sobre regulación de la angiotensina $\|{ }^{138}$. Se ha documentado que posterior a la replica- ción del virus dentro de las células, esta causa daño celular con liberación de citocinas proinflamatorias y activación de la cascada del complemento, lo que permite el reclutamiento de leucocitos, proliferación de linfocitos, liberación de interferón gamma, interleucinas (IL), en especial IL-6, ferritina y factor de necrosis tumoral alfa. Esto se ha relacionado con la activación de macrófagos y consecuente síndrome de activación macofágica con expresión no controlada de citoquinas y liberación del factor activador del plasminógeno, siendo este un posible mecanismo que explicaría la elevación del dímero $D$ y los episodios trombóticos que pueden manifestarse en la piel o de forma sistémica ${ }^{20,23}$.

Los mecanismos de reacciones de hipersensibilidad tipo III o mediadas por inmunocomplejos a antígenos virales los cuales pueden precipitarse y acumularse dentro de los vasos, también forman parte de los principales procesos que conducen a la lesión vascular $^{39}$. La vasculitis, en general, puede afectar vasos pequeños, medianos y grandes; a nivel de la piel se caracteriza por lesiones livedoides hiperémicas o violáceas, con apariencia de red que característicamente afectan las extremidades inferiores, pero también se han reportado lesiones a nivel de tronco. Estas lesiones pueden conducir a necrosis de la epidermis suprayacente y los mecanismos implicados en esta disfunción microvascular pueden incluir la acción directa del virus en las células endoteliales junto a una respuesta inmune alterada ${ }^{40,41}$.

Se ha reportado que las lesiones de livedo reticularis pueden presentarse en cualquier momento de la enfermedad, pero su progresión a vasculitis cutánea papulonecrótica podría indicar complicaciones que conducen a la oclusión vascular, coincidiendo con un aumento en la gravedad de la enfermedad. Así mismo, se han visto asociadas a manifestaciones clínicas como hematuria y posible daño renal ${ }^{9}$, por lo que se considera como el patrón más asociado a mortalidad, en especial en pacientes adultos mayores, con comorbilidades asociadas y en aquellos con formas severas de la enfermedad. Se reporta una incidencia del $6 \%$, en el reporte de series de casos más amplio realizado al momento ${ }^{14}$. En razón de una evaluación más amplia ante la presentación de patrón de livedo o necrosis, se sugiere la realización de estudios de coagulación, conteo de plaquetas, valores de fibrina y dímero $D^{42}$.

\section{Petequias}

La erupción cutánea petequial secundaria a pequeñas hemorragias subdérmicas podría 
considerarse como una manifestación cutánea de enfermedad por COVID-19. Las lesiones petequiales aparecen en cualquier momento durante el curso de la enfermedad; se localizan en el tronco, los glúteos y las extremidades, con tendencia a una distribución flexural o periflexural ${ }^{43,44}$, que característicamente conforman una erupción que no desaparece a la presión ${ }^{17}$. Se han reportado casos donde el exantema petequial en el contexto de enfermedad por COVID-19 puede estar acompañada por trombocitopenia, haciendo una mímica de enfermedad por dengue en zonas endémicas, retrasando el diagnóstico definitivo. Por lo que la infección por SARS-CoV-2 o la posibilidad de coinfección de dengue y la COVID-19, en especial en áreas tropicales donde pueden coexistir arbovirosis y COVID-19. Debe sospecharse ante la evaluación de pacientes con fiebre, sintomatología respiratoria y lesiones petequiales ${ }^{44,45,46}$.

\section{Conclusiones}

En la actualidad, se desconoce mucho sobre la COVID-19; por lo tanto, las manifestaciones dermatológicas observadas en pacientes con COVID-19, constituyen un hallazgo clínico de importancia que amplía el espectro del conocimiento de las manifestaciones clínicas asociadas a esta enfermedad. Los patrones de lesiones cutáneas descritas pueden apoyar el diagnóstico temprano de infección por SARS-Cov-2 y ampliar el espectro de diagnósticos diferenciales a considerar, dentro de los cuales destacan las coinfecciones por virus endémicos o reacciones secundarias a medicamento, debido a que no se puede excluir que en algunos pacientes los hallazgos cutáneos observados pueden representar reacciones cutáneas a los numerosos tratamientos utilizados para COVID-19.

Las manifestaciones cutáneas por COVID-19, a pesar de no tener un valor predictivo certero sobre la estratificación de gravedad de la enfermedad, pueden aportar datos que apoyen la sospecha de un mayor riesgo de complicaciones asociadas. En algunos casos los reconocimientos de las lesiones cutáneas descritas pueden ser de posible ayuda en la toma de medidas de prevención de contagio en pacientes paucisintomáticos o asintomáticos que presenten manifestaciones cutáneas asociadas a COVID-19 sin diagnóstico por pruebas de laboratorio, mostrando ventajas operacionales en el posible contexto de disponibilidad baja de pruebas de laboratorio, con el fin de brindar un tratamiento oportuno y limitar el contagio. La relación entre la infección por
COVID-19 y el desarrollo de manifestaciones cutáneas aún necesita una mayor investigación y más evidencias clínicas.

\section{Agradecimiento}

Expresamos nuestro profundo agradecimiento a las personas que realizaron la revisión del manuscrito.

\section{Financiamiento}

El financiamiento para la realización del manuscrito fue por cuenta propia.

\section{Referencias bibliográficas}

1. Organización Panamericana de la Salud. La OMS caracteriza a COVID-19 como una pandemia. OPS/OMS. 2020. Fecha de consulta: 6 de Agosto de 2020. Disponible en: https://bit.ly/3fdZQBK

2. Van Doremalen N, Bushmaker T, Morris DH, Holbrook MG, Gamble A, Williamson BN, et al. Aerosol and Surface Stability of SARSCoV-2 as Compared with SARS-CoV-1. N. Engl. J. Med. 2020;382(16):1564-1567. DOI: 10.1056/NEJMc2004973

3. Huang C, Wang Y, Li X, Ren L, Zhao J, Hu Y, et al. Clinical features of patients infected with 2019 novel coronavirus in Wuhan, China. The Lancet. 2020;395(10223):497-506. DOI: 10.1016/S0140-6736(20)30183-5

4. Wang D, Hu B, Hu C, Zhu F, Liu X, Zhang J, et al. Clinical Characteristics of 138 Hospitalized Patients With 2019 Novel CoronavirusInfected Pneumonia in Wuhan, China. JAMA. 2020;323(11):1061. DOI: 10.1001/ jama.2020.1585

5. Freeman EE, McMahon DE, Fitzgerald ME, Fox LP, Rosenbach M, Takeshita J, et al. The American Academy of Dermatology COVID-19 registry: Crowdsourcing dermatology in the age of COVID-19. J. Am. Acad. Dermatol. 2020;83(2):509-510. DOI: 10.1016/j.jaad.2020.04.045

6. Drago F, Ciccarese G, Gasparini G, Cogorno L, Javor S, Toniolo A, et al. Contemporary infectious exanthems: an update. Future Microbiol. 2017;12(2):171-193. DOl:10.2217/ fmb-2016-0147

7. Wang K, Chen W, Zhou Y-S, Lian J-Q, Zhang Z, Du P, et al. SARS-CoV-2 invades host cells via a novel route: CD147spike protein. Microbiology; 2020. DOI: 10.1101/2020.03.14.988345

8. Hamming I, Timens W, Bulthuis M, Lely A, Navis G, Van Goor H. Tissue distribution of ACE2 protein, the functional receptor for SARS coronavirus. A first step in understanding SARS pathogenesis. J. 
Pathol. 2004;203(2):631-637. DOI: 10.1002/ path.1570

9. Potekaev NN, Zhukova OV, Protsenko DN, Demina OM, Khlystova EA, Bogin V. Clinical characteristics of dermatologic manifestations of COVID-19 infection: case series of 15 patients, review of literature, and proposed etiological classification. Int. J. Dermatol. 2020;59(8):1000-1009. DOI: 10.1111/ijd.15030

10. Kaya G, Kaya A, Saurat J-H. Clinical and histopathological features and potential pathological mechanisms of skin lesions in COVID-19: review of the literature. Dermatopathology. 2020;7(1):3-16. DOI: 10.3390/dermatopathology7010002

11. World Health Organization. Criteria for releasing COVID-19 patients from isolation. Geneva: World Health Organization; 2020. 5 p. Disponible en:WHO-2019-nCoV-Sci_BriefDischarge_From_Isolation-2020.1-eng.pdf

12. The RECOVERY Collaborative Group. Dexamethasone in hospitalized patients with Covid-19 - preliminary report. N. Engl. J. Med. 2021;384(8):693-704. DOI: 10.1056/ NEJMoa2021436

13. Fahmy DH, El-Amawy HS, El-Samongy MA, Fouda AA, Soliman SH, El-Kady A, et al. COVID-19 and dermatology: a comprehensive guide for dermatologists. J. Eur. Acad. Dermatol. Venereol. 2020;34(7):1388-1394. DOI: 10.1111/ jdv.16545

14. Galván Casas C, Català A, Carretero Hernández G, Rodríguez-Jiménez $P_{\text {, }}$ Fernández-Nieto D, Rodríguez-Villa Lario A, et al. Classification of the cutaneous manifestations of COVID-19: a rapid prospective nationwide consensus study in Spain with 375 cases. Br. J. Dermatol. 2020;183(1):71-77. DOI: 10.1111/bjd.19163

15. Rahimi H, Tehranchinia Z. A comprehensive review of cutaneous manifestations associated with COVID-19. BioMed Res. Int. 2020;2020:1-8. DOI: 10.1155/2020/1236520

16. Yan G, Lee CK, Lam LTM, Yan B, Chua YX, Lim AYN, et al. Covert COVID-19 and falsepositive dengue serology in Singapore. Lancet Infect. Dis. 2020;20(5):536. DOI: 10.1016/S1473-3099(20)30158-4

17. Ortega-Quijano D, Jimenez-Cauhe J, Selda-Enriquez G, Fernandez-Guarino M, Fernandez-Nieto D. Algorithm for the classification of COVID-19 rashes. J. Am. Acad. Dermatol. 2020;83(2):e103-e104. DOl: 10.1016/j.jaad.2020.05.034

18. Piccolo V, Neri I, Filippeschi C, Oranges T, Argenziano G, Battarra VC, et al. Chilblainlike lesions during COVID-19 epidemic: a preliminary study on 63 patients. J. Eur. Acad. Dermatol. Venereol. 2020;34(7). DOl: 10.1111/jdv.16526
19. Le Cleach L, Dousset L, Assier H, Fourati S, Barbarot S, Boulard C, et al. Most chilblains observed during the COVID-19 outbreak occur in patients who are negative for COVID-19 on polymerase chain reaction and serology testing*. Br. J. Dermatol. 2020;183(5):866-874. DOI: 10.1111/ bjd. 19377

20. Colonna C, Monzani NA, Rocchi A, Gianotti R, Boggio F, Gelmetti C. Chilblain-like lesions in children following suspected COVID-19 infection. Pediatr. Dermatol. 2020;37(3):437440. DOI: 10.1111/pde.14210

21. Freeman EE, McMahon DE, Lipoff JB, Rosenbach M, Kovarik C, Takeshita J, et al. Pernio-like skin lesions associated with COVID-19: a case series of 318 patients from 8 countries. J. Am. Acad. Dermatol. 2020;83(2):486-492. DOI: 10.1016/j. jaad.2020.05.109

22. Zhang Y, Xiao M, Zhang S, Xia P, Cao W, Jiang W, et al. Coagulopathy and antiphospholipid antibodies in patients with Covid-19. N. Engl. J. Med. 2020;382(17):e38. DOI: 10.1056/ NEJMc2007575

23. González González F, Cortés Correa C, Peñaranda Contreras E. Manifestaciones cutáneas en pacientes con COVID-19: características clínicas y mecanismos fisiopatológicos postulados. Actas DermoSifiliográficas. 2021;112(4):314-323. DOI: 10.1016/j.ad.2020.11.013

24. Fernandez-Nieto D, Ortega-Quijano D, Jimenez-Cauhe J, Burgos-Blasco P, PerosanzLobo D, Suarez-Valle A, et al. Clinical and histological characterization of vesicular COVID-19 rashes: a prospective study in a tertiary care hospital. Clin. Exp. Dermatol. 2020;45(7):872-875. DOI: 10.1111/ced.14277

25. Sachdeva M, Gianotti R, Shah M, Bradanini L, Tosi D, Veraldi S, et al. Cutaneous manifestations of COVID-19: Report of three cases and a review of literature. J. Dermatol. Sci. 2020;98(2):75-81. DOI: 10.1016/j. jdermsci.2020.04.011

26. Marzano AV, Cassano N, Genovese G, Moltrasio C, Vena GA. Cutaneous manifestations in patients with COVID-19: a preliminary review of an emerging issue. Br. J. Dermatol. 2020;183(3):431-442. DOI: 10.1111/bjd.19264

27. Gisondi P, Plaserico S, Bordin C, Alaibac M, Girolomoni G, Naldi L. Cutaneous manifestations of SARS-CoV-2 infection: a clinical update. J. Eur. Acad. Dermatol. Venereol. 2020;34(11):2499-2504. DOI: 10.1111/jdv.16774

28. Amatore F, Macagno N, Mailhe M, Demarez B, Gaudy-Marqueste C, Grob JJ, et al. SARSCoV-2 infection presenting as a febrile rash. J. Eur. Acad. Dermatol. Venereol. 2020;34(7). DOI: $10.1111 / j d v .16528$ 
29. Henry D, Ackerman M, Sancelme E, Finon A, Esteve E. Urticarial eruption in COVID-19 infection. J. Eur. Acad. Dermatol. Venereol. 2020;34(6). DOI: 10.1111/jdv.16472

30. Hassan K. Urticaria and angioedema as a prodromal cutaneous manifestation of SARS-CoV-2 (COVID-19) infection. BMJ Case Rep. 2020;13(7):e236981. DOI: 10.1136/bcr2020-236981

31. Daneshgaran G, Dubin DP, Gould DJ. Cutaneous Manifestations of COVID-19: An Evidence-Based Review. Am. J. Clin. Dermatol. 2020;21(5):627-639. DOI: 10.1007/ s40257-020-00558-4

32. Morey-Olivé M, Espiau M, Mercadal-Hally M, Lera-Carballo E, García-Patos V. Cutaneous manifestations in the current pandemic of coronavirus infection disease (COVID 2019). An. Pediatría Engl. Ed. 2020;92(6):374-375. DOI: 10.1016/j.anpede.2020.04.002

33. Ehsani AH, Nasimi M, Bigdelo Z. Pityriasis rosea as a cutaneous manifestation of COVID-19 infection. J. Eur. Acad. Dermatol. Venereol. 2020;34(9). DOI: 10.1111/jdv.16579

34. Dursun R, Temiz SA. The clinics of HHV -6 infection in COVID -19 pandemic: Pityriasis rosea and Kawasaki disease. Dermatol. Ther. 2020;33(4). DOI: 10.1111/dth.13730

35. Kutlu Ö, Metin A. Relative changes in the pattern of diseases presenting in dermatology outpatient clinic in the era of the COVID-19 pandemic. Dermatol. Ther. 2020;33(6). DOI: 10.1111/dth.14096

36. Criado PR, Abdalla BMZ, de Assis IC, Van Blarcum de Graaff Mello C, Caputo GC, Vieira IC. Are the cutaneous manifestations during or due to SARS-CoV-2 infection/COVID-19 frequent or not? Revision of possible pathophysiologic mechanisms. Inflamm. Res. 2020;69(8):745-756. DOI: 10.1007/ s00011-020-01370-w

37. Avellana Moreno R, Estela Villa LM, Avellana Moreno V, Estela Villa C, Moreno Aparicio MA, Avellana Fontanella JA. Cutaneous manifestation of COVID-19 in images: a case report. J. Eur. Acad. Dermatol. Venereol. 2020;34(7). DOI: 10.1111/jdv.16531

38. Senchenkova EY, Russell J, Yildirim A, Granger DN, Gavins FNE. Novel Role of T
Cells and IL-6 (Interleukin-6) in Angiotensin II-Induced Microvascular Dysfunction. Hypertension. 2019;73(4):829-838. DOI: 10.1161/HYPERTENSIONAHA.118.12286

39. Millikan LE, Flynn TC. Infectious etiologies of cutaneous vasculitis. Clin. Dermatol. 1999;17(5):509-514. DOI: 10.1016/S0738081X(99)00061-9

40. Elmas ÖF, Demirbaş A, Özyurt K, Atasoy $M$, Türsen Ü. Cutaneous manifestations of COVID-19: A review of the published literature. Dermatol. Ther. 2020;33(4). DOI: 10.1111/dth.13696

41. Tang N, Li D, Wang X, Sun Z. Abnormal coagulation parameters are associated with poor prognosis in patients with novel coronavirus pneumonia. J. Thromb. Haemost. 2020;18(4):844-847. DOI: 10.1111/ jth. 14768

42. Manalo I. A Dermatologic Manifestation of COVID-19: Transient Livedo Reticularis. 2020.

43. Seirafianpour F, Sodagar S, Pour Mohammad A, Panahi P, Mozafarpoor S, Almasi S, et al. Cutaneous manifestations and considerations in COVID-19 pandemic: A systematic review. Dermatol. Ther. 2020;33(6). DOI: 10.1111/dth.13986

44. Jimenez-Cauhe J, Ortega-Quijano D, Prieto-Barrios M, Moreno-Arrones OM, Fernandez-Nieto D. Reply to "COVID-19 can present with a rash and be mistaken for dengue": Petechial rash in a patient with COVID-19 infection. J. Am. Acad. Dermatol. 2020;83(2):e141-e142. DOI: 10.1016/j. jaad.2020.04.016

45. Cardona-Ospina JA, Arteaga-Livias K, Villamil-Gómez WE, Pérez-Díaz CE, Katterine Bonilla-Aldana D, Mondragon-Cardona Á, et al. Dengue and COVID-19, overlapping epidemics? An analysis from Colombia. J. Med. Virol. 2021;93(1):522-527. DOI: 10.1002/jmv.26194

46. Saavedra-Velasco M, Chiara-Chilet C, Pichardo-Rodriguez R, Grandez-Urbina A, Inga-Berrospi F. Coinfección entre dengue y COVID-19: Necesidad de abordaje en zonas endémicas. Rev. Fac. Cienc. Médicas Córdoba. 2020;77(1):52-54. DOI: 10.31053/1853.0605.v77.n1.28031 